\title{
Patients who develop post-operative atrial fibrillation have reduced survival after off-pump coronary artery bypass grafting
}

\author{
Akhil Ghurram $^{1} \cdot$ Neethu Krishna ${ }^{1} \cdot$ Renjitha Bhaskaran ${ }^{2} \cdot$ Natarajan Kumaraswamy $^{3}$ - Aveek Jayant ${ }^{4}$. \\ Praveen Kerala Varma ${ }^{1}$ [D
}

Received: 28 February 2019 / Revised: 13 May 2019 / Accepted: 31 May 2019 / Published online: 19 July 2019

(C) Indian Association of Cardiovascular-Thoracic Surgeons 2019

\begin{abstract}
Objective Post-operative atrial fibrillation (POAF) increases hospital stay, resource utilization, morbidity, and mortality. However, there is paucity of data about its effect in Indian patients undergoing off-pump coronary artery bypass grafting (CABG).

Methods Seven hundred forty-eight patients underwent off-pump CABG from January 2015 to December 2016 (24 months). One hundred twenty-seven patients (16.7\%) developed POAF. In an effort to mitigate the effects of wider risk factors on perioperative outcomes, a separate sub-analysis of patients based on risks quantified by EuroSCORE II $(<>3)$ was also performed.

Results Age $>60$ years and development of sepsis were the independent predictors for the development of POAF. Thirty-day/ mortality rate was higher in the POAF group $(7.1 \%$ vs. $1.4 \% ; p$ value $<0.001)$. POAF was associated with increased ICU and hospital stay and increased incidence of stroke and renal dysfunction. The survival was significantly lower in the POAF group compared with the normal sinus rhythm (NSR) (3-year survival in POAF was $81.3 \%$ vs. 94.4\% in the NSR group; Hazard ratio (HR) 3.867 (1.989-7.516)). Intra-aortic balloon pump (IABP) usage, age $\geq 60$ years and sepsis were independent predictors for the development of POAF in low-risk patients. For the NSR group, 1-year survival was $98 \%$ and 3 -year survival was $95.7 \%$. For the POAF group, 1-year survival was $94.4 \%$ and 3-year survival was $84.0 \%$ (HR. 3.794 (1.897-7.591)).

Conclusion The incidence of POAF was lower than reported in the wider global literature. Increasing age and development of post-operative sepsis were strong independent predictors of POAF. POAF increases the morbidity; length of hospital stay and these patients show decreased survival after off-pump CABG.
\end{abstract}

Keywords Post-operative atrial fibrillation · Atrial fibrillation · Off-pump CABG

\section{Background}

Atrial fibrillation (AF) is the most common arrhythmia after cardiac surgery [1]. Many risk factors exist for development of

Praveen Kerala Varma

varmapk@gmail.com

1 Department of Cardiothoracic Surgery, Amrita Institute of Medical Sciences, Amrita Vishwa Vidyapeetham (Amrita University), Kochi, India

2 Department of Biostatistics, Amrita Institute of Medical Sciences, Amrita Vishwa Vidyapeetham (Amrita University), Kochi, India

3 Department of Cardiology, Amrita Institute of Medical Sciences, Amrita Vishwa Vidyapeetham (Amrita University), Kochi, India

4 Department of Anesthesiology, Amrita Institute of Medical Sciences, Amrita Vishwa Vidyapeetham (Amrita University), Kochi, India post-operative AF (POAF) [2-5]. POAF is the consequence of multiple re-entry wavelets resulting from the "dispersion of atrial refractoriness" (non-uniformity of the distribution of local atrial refractory periods). Dispersion of atrial refractoriness requires an atrial structural substrate. It might be the consequence of advanced age, hypertension, diabetes, obesity, left atrial enlargement, and left ventricular hypertrophy. Operative and postoperative factors such as atrial ischemia and surgical atrial injury might also contribute to the development of the structural substrate. Inflammation, oxidative stress, and genetic factors also seem to play an important role. Once these conditions are present, a triggering factor such as an atrial premature contraction, electrolyte imbalance, and/or enhanced adrenergic or vagal stimulation will initiate POAF [6]. Both experimental and clinical studies have provided strong evidence that atrial ischemia is the primary stimulus that triggers the development of POAF in patients with atrial "dispersion of refractoriness" [6]. Since its 
incidence peaks at post-operative day 2 or 3 , inflammatory mechanisms, both systemic and local, may also play an important role in the development of POAF [2].

Off-pump technique of coronary artery bypass grafting $(\mathrm{OPCAB})$ is believed to elicit less systemic inflammation than on-pump surgery because of reduced cytokine responses and less myocardial injury. OPCAB also allows avoidance of ischemia/reperfusion injury and reduces the oxidative stress. Ascione et al. [7] reported that the incidence of POAF after CABG was decreased, from $45 \%$ in patients operated under cardiopulmonary bypass (CPB) to $11 \%$ in those operated without CPB. Another study [8] documented reduction in POAF, from $28 \%$ in hearts protected with cardioplegic arrest to $9 \%$ in those operated on using intermittent ischemia. Both these studies point to inadequate atrial protection using cardioplegic arrest as the trigger for developing POAF.

The incidence of POAF also varies depending upon the race. In a study of Indian patients undergoing $C A B G$ under $\mathrm{CPB}$ and cardioplegic arrest, Borde et al. [9] found the incidence of POAF to be $13 \%$, much lower than reported in western literature. In one trans-continental study conducted in 70 centers [10], the incidence of POAF after CABG surgery was $32.3 \%$. This incidence was similar among patients in the USA (33.7\%), Canada (36.6\%), Europe (34.0\%), the UK (31.6\%), and the Middle East (41.6\%), but the incidence was lower in South America (17.4\%) and Asia (15.7\%).

In the past, POAF was considered as a benign arrhythmia [11]; multiple studies [12-15] have shown that POAF increases the post-operative morbidity and mortality in the short term and has long-term consequences as well. After adjusting for the influence of baseline patient operative features, surgeon and hospital variability, and operative era, LaPar and associates found that POAF was associated with a 1.8 -fold to twofold increase in the odds of mortality for isolated CABG and aortic valve procedures. They also found that patients suffering POAF had twofold to fourfold increase in stroke, greater hospital resource utilization, and increased costs [16].

However, there is paucity of data in literature about its effect in off-pump CABG (OPCAB) patients from the Indian subcontinent, where more than $60 \%$ of patients undergo OPCAB [17]. Hence, there is a pressing need to evaluate the incidence, risk factors, and effect of POAF in $\mathrm{OPCAB}$ patients from India.

\section{Objective}

The aims of our study were

1. To determine the incidence of POAF after OPCAB surgery in Indian patients
2. To find the risk factors for development of POAF after OPCAB surgery and

3. To answer the question whether POAF increases the mortality and morbidity after OPCAB surgery.

\section{Materials and methods}

From January 2015 to December 2016, consecutive patients who underwent OPCAB (748 patients) were enrolled in the study. Patients were divided into two groups based on their post-operative rhythm status: AF $(n=127 ; 16.7 \%)$ and NSR $(n=621 ; 83.3 \%)$. Pre-operative variables included in the study were age, gender, urgency of surgery, diabetes mellitus, hypertension, dyslipidemia, chronic obstructive pulmonary disease (COPD), peripheral vascular disease (PVD), carotid stenosis, pre-operative myocardial infarction, renal dysfunction, EuroSCORE II, and left ventricular ejection fraction. Post-operative variables such as ventilation hours, stroke, renal failure, IABP usage, usage of inotropes, ICU stay, length of hospital stay, and mortality were included. Data was captured prospectively and analyzed using an electronic institutional database.

Operative risk is a potentially confounding variable in any analysis of POAF. To reduce its impact, we did a sub-analysis of patients with low operative risk (EuroSCORE II $\leq 3$ ).

Exclusion criteria $\mathrm{CABG}$ performed using cardiopulmonary bypass ( 25 patients) were excluded from the study. Patients with pre-operative AF were also excluded from the study.

Definition of variables POAF was defined by the Society of Thoracic Surgery (STS) definition - detection of AF or atrial flutter requiring treatment, prior to hospital discharge. Sepsis was defined as post-operative rise in temperature above $38.6{ }^{\circ} \mathrm{C}$ with white cell count $>12,000$ or $<4000$ with the need for starting empirical intra-venous antibiotics (STS definition). Usage of inotropes was defined as any requirement of inotropes or vasopressor in ICU. Pre-operative renal dysfunction was defined as creatinine clearance of less than $85 \mathrm{ml} / \mathrm{min}$ (EuroSCORE II criteria). Post-operative renal dysfunction was defined using the Acute Kidney Injury Network (AKIN) criteria [18].

Ethical committee approval The study protocol was approved by the Institutional Ethical Committee (IRB-AIMS-2019004). The need for informed consent was waived since the data used in this study had already been collected for the surgical database registry maintained by our Department of Cardiothoracic and vascular Surgery. All personal identifying data was removed from the study database so that individuals could not be identified. 
OPCAB technique All patients underwent median sternotomy. Anterior fat pad was preserved in all patients. Heparin was administered to achieve a target-activated clotting time of more than $400 \mathrm{~s}$. Left internal mammary artery was the preferred conduit to bypass left anterior descending artery. Saphenous vein grafts were the preferred conduit to bypass the other systems. Proximal anastomoses were constructed in ascending aorta using partial occlusion clamp. Posterior pericardiotomy was not performed in any of the patients. Heparin was fully reversed with protamine after the procedure. Patients were shifted to dedicated intensive care unit for post-operative monitoring.

Perioperative care Angiotensin-converting enzyme (ACE) inhibitors, $\beta$-blocker therapy, and aspirin were continued to the day of surgery. Clopidogrel was withdrawn 2 days prior to operation. $\beta$-Blocker was started on post-operative day 1 , if the patients were not on inotropes and vasopressor agents. All patients received atorvastatin $20 \mathrm{mg}$ on the first post-operative day. Hypokalemia below $3.5 \mathrm{mEq} / \mathrm{L}$ was corrected. Routine magnesium sulfate correction was not done in any patients. All patients were monitored with continuous cardiac output with pulmonary artery flotation catheter (Edward Lifesciences, USA). POAF was controlled by amiodarone infusion if hemodynamically stable. Hemodynamically unstable patients underwent cardio-version. Patients with recurrent POAF and persisting AF were anticoagulated by warfarin to a target international normalized ratio of 2.0 , for 1 month. If the repeat electrocardiogram (ECG) showed normal sinus rhythm, anticoagulation was stopped. All patients not on warfarin were treated with dual antiplatelet therapy (aspirin $150 \mathrm{mg}+$ clopidogrel $75 \mathrm{mg}$ ) for 1 year post-CABG; thereafter, only aspirin was prescribed.

Follow-up All discharged-from-hospital patients were evaluated in our outpatient clinic at 30 days. Then, the patients were asked to follow-up at 3 months after surgery and then yearly. They were evaluated with chest X-ray and ECG. All patients with left ventricular (LV) ejection fraction $\leq 35 \%$ underwent echocardiography evaluation at each follow-up. Patients who did not turn up for follow-up (124 patients) were contacted telephonically in February 2018. Twenty-four patients (3.2\%) were lost to follow-up. Median follow-up period was 630 days. Data were captured prospectively and entered in to a database.

Statistical analysis Analysis was performed using IBM SPSS 20.0 version (SPSS Inc., Chicago, USA). The continuous variables were presented using mean \pm standard deviation (SD). The categorical variables were expressed using frequency and percentage. To test the statistical significance of the difference in percentages of categorical variables between the two groups (AF and NSR), the chi-square test was used. To test the statistical significance of the difference in the mean of continuous variables between the two groups, the independent sample $t$ test was used. Multivariate analysis (binary logistic regression) was used to find the most significant variables. All the variables with $p$ value $<0.2$ in the univariate analysis were included for multivariate analysis. Kaplan-Meier analysis was used to find the survival probability and comparison was done using log-rank test. A $p$ value $<0.05$ was considered as statistically significant.

\section{Results}

99.2\% of POAF developed in patients above the age of 50 years ( $p$ value of 0.002 ). In univariate analysis of preoperative variables, increasing age ( $p$ value $<0.001)$, emergency surgery ( $p$ value 0.001 ), pre-operative renal dysfunction ( $p$ value 0.008 ), pre-operative myocardial infarction ( $p$ value 0.012 ), low left ventricular ejection fraction ( $p$ value 0.001 ), IABP usage ( $p$ value $<0.001$ ), development of post-operative sepsis ( $p$ value $<0.001$ ), blood stream infection ( $p$ value $<$ 0.001 ), and EuroSCORE II value more than 3 ( $p$ value $<$ 0.001 ) were identified as risk factors for POAF. Multivariate analysis is given in Table 1 .

The ventilator hours and inotropes usage were significantly higher in the POAF group with $p$ value $<0.001$. The incidence of post-operative stroke and renal dysfunction was also higher in POAF patients. The length of ICU stay and hospital stay was significantly higher in the AF group (Table 2). Thirty-day/ in-house mortality rate was also highly significant in the POAF group (7.1\% vs. $1.5 \%$; $p$ value $<0.001$ ) (Table 2).

For the NSR group, 1-year survival was $97.3 \%, 2$-year survival was $96.9 \%$, and 3-year survival was $94.4 \%$. For the POAF group, 1-year survival was $88.7 \%$ and 3-year survival was $81.3 \%$. In overall survival analysis, the mean survival time of the NSR group was $1110.07 \pm 8.13$ days and the mean survival time of the POAF group was $997.62 \pm 34.71$ days which showed statistically significant difference in the survival probability with $p$ value $<0.001$. The HR was 3.867 (1.997.52) (Fig. $1 \mathrm{~A}, \mathrm{~B}$ ). The mean survival time of the NSR group

Table 1 Multivariate analysis

\begin{tabular}{llll}
\hline Variables & Wald & $p$ value & OR (95\% of CI) \\
\hline Age & & & \\
$<50$ & 19.627 & $<0.001^{* *}$ & 1 \\
$50-60$ & 2.680 & 0.102 & $5.45(0.72-41.56)$ \\
$60-70$ & 5.384 & $0.02^{*}$ & $10.79(1.45-80.50)$ \\
$\geq 70$ & 7.506 & $0.006^{*}$ & $17.05(2.24-129.73)$ \\
Sepsis & 41.567 & $<0.001^{* *}$ & $5.32(3.20-8.85)$ \\
IABP used & 3.409 & 0.065 & $4.39(0.91-21.08)$ \\
\hline
\end{tabular}

Significant $p$ value $<0.05^{*}$ and $<0.001 * *$

$O R$ odds ratio, $C I$ confidence interval, $I A B P$ intra-aortic balloon pump 
Table 2 Comparison of outcome variables with post-operative normal sinus rhythm and atrial fibrillation

\begin{tabular}{|c|c|c|c|c|}
\hline Variables & $\begin{array}{l}\text { NSR }(n(\%)) \\
621(\%)\end{array}$ & $\begin{array}{l}\operatorname{AF}(n(\%)) \\
127(\%)\end{array}$ & $\begin{array}{l}\text { Univariate analysis } \\
\text { OR ( } 95 \% \text { of } \mathrm{CI})\end{array}$ & $p$ value \\
\hline Ventilation hours & $11.93 \pm 14.114$ & $31.31 \pm 75.434$ & $1.02(1.01-1.03)$ & $<0.001 * *$ \\
\hline Duration of inotropes & $1.50 \pm 1.34$ & $3.31 \pm 3.77$ & $1.56(1.34-1.82)$ & $<0.001 * *$ \\
\hline Post-op stroke(Y) & $5(0.8)$ & $4(3.1)$ & $4.01(1.06-15.13)$ & $0.027 *$ \\
\hline Post-op renal dysfunction(Y) & $5(0.8)$ & $4(3.1)$ & $4.01(1.06-15.13)$ & $0.027 *$ \\
\hline Length of ICU stay & $3.07 \pm 1.90$ & $6.15 \pm 5.40$ & $1.51(1.34-1.69)$ & $<0.001 * *$ \\
\hline Length of hospital stay & $10.81 \pm 4.60$ & $15.8 \pm 10.87$ & $1.10(1.07-1.13)$ & $<0.001 * *$ \\
\hline \multicolumn{5}{|l|}{ 30-day mortality } \\
\hline Alive & $612(98.5)$ & $118(92.9)$ & $5.19(2.02-13.34)$ & $<0.001 * *$ \\
\hline Dead & $9(1.5)$ & $9(7.1)$ & & \\
\hline
\end{tabular}

Significant $p$ value $<0.05 *$ and $<0.001 * *$

$n$ number of patients, $N S R$ normal sinus rhythm, $A F$ atrial fibrillation, post-op post-operative, $I C U$ intensive care unit, $O R$ odds ratio, $C I$ confidence interval above 30 days was $1123.07 \pm 6.58$ and the mean survival time of the POAF above 30 days was $1075.15 \pm 26.08$ which showed statistically significant difference in the survival probability with $p$ value $<0.001$. Eighteen (12 patients in the NSR group and 6 in the POAF group) patients expired during follow-up. In 2 patients (one in each group), the cause of death could not be ascertained. All patients in POAF and 8 patients in NSR died due to cardiac cause. The non-cardiac causes included COPD exacerbation in 2 patients, malignancy in one patient, and sepsis from cellulites in another patient. There was no difference in myocardial infarction rates, angina rates, repeat revascularization, and heart failure admissions between the 2 groups. However, late occurrence of chronic kidney disease and stroke was more common in the POAF group during follow-up (Table 3).

In 748 patients, 529 patients in the NSR group and 92 patients in the AF group were low-risk patients (EuroSCORE II <3) included for a separate sub-analysis of this cohort. In the univariate analysis of low-risk patients, age

Table 3 Follow-up

\begin{tabular}{lll}
\hline Variables & \multicolumn{2}{l}{ Alive $(n=706)$} \\
\cline { 2 - 3 } & $\begin{array}{ll}\operatorname{NSR}(n(\%)) \\
591(\%)\end{array}$ & $\begin{array}{l}\mathrm{AF}(n(\%)) \\
115(\%)\end{array}$ \\
\hline Revascularization & $10(1.69)$ & $2(1.74)$ \\
Stroke & $3(0.51)$ & $2(1.74)$ \\
Heart failure & $4(0.68)$ & $0(0)$ \\
Renal dysfunction & $18(3.05)$ & $8(6.96)$ \\
Renal failure requiring dialysis & $4(0.68)$ & $0(0)$ \\
Myocardial infarction & $5(0.85)$ & $0(0)$ \\
Angina & $11(1.86)$ & $2(1.74)$ \\
Mid-term mortality & $12(2.03)$ & $6(5.23)$ \\
\hline
\end{tabular}

NSR normal sinus rhythm, $A F$ atrial fibrillation, $n$ number of patients
( $p$ value 0.001 ), pre-operative renal dysfunction ( $p$ value 0.016 ), development of post-operative sepsis ( $p$ value < $0.001)$, and blood stream infections (BSI) ( $p$ value 0.004$)$ were identified as risk factors for POAF. Multivariate analysis is given in Table 4.

The ventilator hours and inotrope usage were highly significant in the POAF group with $p$ value $<0.001$. Postoperative stroke showed significance in the POAF group with $p$ value 0.036 . The length of ICU stay and hospital stay was statistically highly significant in the POAF group with $p$ value $<0.001$ (Table 5). Thirty-day/in-house mortality rate was also highly significant in the POAF group $(4.3 \%$ vs. $0.8 \%$, HR 5.966; confidence interval (CI) $1.47-24.29, p$ value 0.005 ) (Table 5).

The stroke rate and incidence of renal dysfunction were higher during follow-up in the POAF group (Table 6). The overall survival analysis was done in low-risk patients of the NSR and AF groups with mean survival time $1113.26 \pm 7.85$. For the NSR group, 1-year survival was $98 \%$ and 3-year survival was $95.7 \%$. For the POAF group, 1-year survival was $94.4 \%$ and 3 -year survival was $84.0 \%$. The mean survival time of the NSR group was $1120.91 \pm 7.42$ days and the mean survival time of the POAF group was $1053.49 \pm 34.38$ days which showed statistically significant difference in the survival probability with $p$ value 0.02 . The hazard ratio was 3.79 (1.90-7.59) (Fig. 2 A, B).

Of 118 patients in the POAF group discharged alive, 20 patients $(16.9 \%)$ were in AF. Ninety-eight patients $(83 \%)$ were on oral amiodarone. At 30 days, 14 patients $(11.7 \%)$ were in AF. Persistent AF after 3 months was seen in 3 patients $(2.5 \%)$. Cardio-version was attempted in all the three patients; one patient reverted to NSR. Remaining 2 patients remained in chronic AF; out of which, one patient expired after 31 months from severe LV dysfunction, renal failure, and urosepsis. 
Fig. 1 A and B Kaplan-Meir estimate of survival of patients in all patients; NSR vs. POAF

Survival Curve and Hazard Function

a

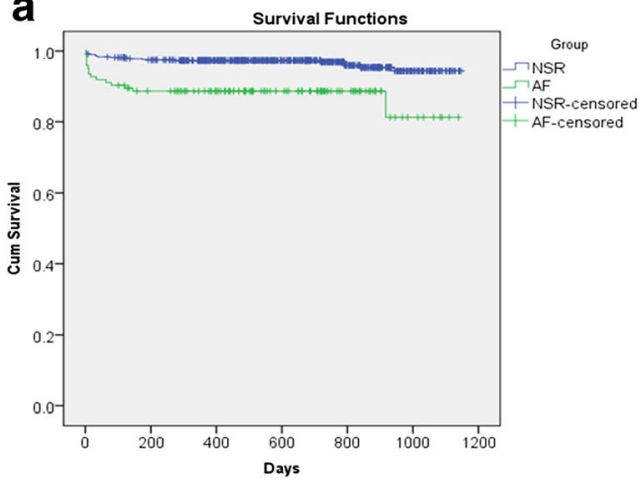

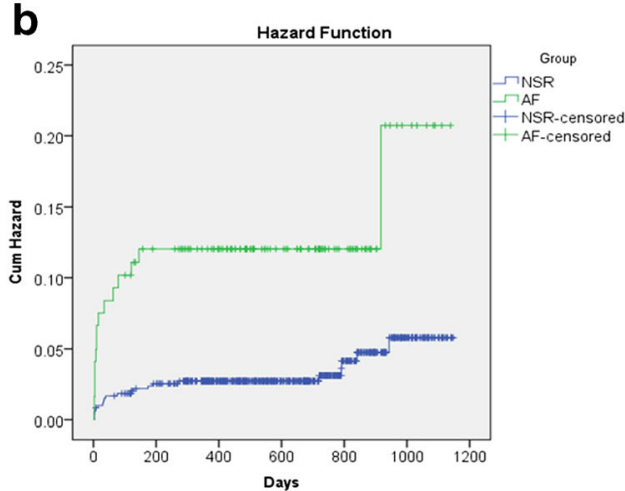

\section{Discussion}

The study showed that (1) the incidence of POAF was much lower than reported in western literature, (2) sicker patients have more propensities to develop AF, (3) the patients who developed POAF had much higher short-term mortality and morbidity, and (4) they had lower survival chance when compared with NSR patients in follow-up.

The incidence of POAF was $16.7 \%$ in this study. New onset of AF occurred in $24.9 \%$ of patients after CABG according to the latest STS data report [19]. The role of $\mathrm{OPCAB}$ in reducing the incidence of AF is controversial. CPB inclusive of cardioplegic arrest was the main independent predictor of POAF (OR 7.4, CI 3.4-17.9) in patients undergoing $\mathrm{CABG}$ in a prospective randomized study reported by Ascione et.al [7]. However, this report was criticized for its small patient sample size and singlecenter origin. Moreover, several studies failed to show statistical association between OPCAB and a lower incidence of POAF. Investigators of the ROOBY trial (the randomized on vs. off bypass trial) found that the

Table 4 Multivariate analysis in low-risk patients

\begin{tabular}{|c|c|c|c|}
\hline Variables & Wald & $p$ value & OR $(95 \%$ of $\mathrm{CI})$ \\
\hline IABP used & 4.76 & $0.029 *$ & $34.43(1.43-826.90)$ \\
\hline \multicolumn{4}{|l|}{ Age } \\
\hline$<50$ & 14.82 & $0.002 *$ & 1 \\
\hline $50-60$ & 2.70 & 0.101 & $6.35(0.70-57.64)$ \\
\hline $60-70$ & 5.56 & $0.018 *$ & $13.96(1.56-124.88)$ \\
\hline$\geq 70$ & 6.46 & $0.011 *$ & $18.24(1.94-171.20)$ \\
\hline Sepsis & 26.06 & $<0.001 * *$ & $5.60(2.89-10.86)$ \\
\hline Pre-op recent MI & 3.51 & 0.061 & $0.62(0.38-1.02)$ \\
\hline Pre-op renal dysfunction & 2.99 & 0.084 & $0.47(0.20-1.11)$ \\
\hline
\end{tabular}

Significant $p$ value $<0.05^{*}$ and $<0.001 * *$

$O R$ odds ratio, $C I$ confidence interval, $I A B P$ intra-aortic balloon pump, pre-op pre-operative, $M I$ myocardial infarction incidence of POAF was $27 \%$ in off-pump group with no difference in POAF rate based on off-pump vs. on-pump strategy of revascularization [20]. The ROOBY trial was criticized for the fact that the conversion rate to $\mathrm{CPB}$ was unacceptably high at $12 \%$ and has drawn skepticism as to the level of "off pump" experience of the surgeons involved in the study. Patients enrolled were almost exclusively males and there was a trend toward enrolling lower-risk patients and excluding higher-risk patients. It is questionable whether this study is applicable to the real world. In the mass III trial, the incidence of POAF in OPCAB patients was $35 \%$ and much higher than patients undergoing on-pump CABG [21]. Since we rarely perform $\mathrm{CABG}$ using $\mathrm{CPB}$, we are unable to comment on this outlier study.

The average age in our study was 62 years \pm 10 which is lower than those reported from the western data [19]. Moreover, in patients below the age of 50 years who underwent $\mathrm{CABG}$ (which formed $8.7 \%$ of our cohort), incidence of POAF was only $0.8 \%$. In the recently published OPERA (omega-3 fatty acids for prevention of post-operative atrial fibrillation) trial [22], compared with on-pump CABG, OPCAB was significantly associated with a lower risk of POAF (odds ratio (OR), 0.53; 95\% CI, 0.33-0.85) among men and in patients aged less than 65 years (OR, 0.35; 95\%CI, 0.16-0.75). Lower POAF rates are reported in the south Asian population $[9,10]$ compared with the Caucasian race. Younger age at the time of revascularization and lower body mass index are the usual reasons for the lower incidence of POAF in the south Asian population [23]. Further, data from the Asian continent is not as widely reported in the global literature for want of systemic data collection and reportage. Owing to the purported differences of race as seen above, it is likely that the outcomes from Asia will differ from those reported in the North American and European continents.

Advanced age is associated with degenerative and inflammatory modifications in atrial anatomy (dilation, fibrosis), 
Table 5 Comparison of outcome variables of low-risk patients (EuroSCORE II <3) with postoperative normal sinus rhythm and atrial fibrillation

\begin{tabular}{lllll}
\hline Variables & NSR $(n(\%))$ & AF $(n(\%))$ & & Univariate analysis \\
\cline { 4 - 5 } & $529(\%)$ & $92(\%)$ & OR $(95 \%$ of CI $)$ & $p$ value \\
\hline Ventilation hours & $11.21 \pm 13.52$ & $17.54 \pm 23.37$ & $1.02(1.01-1.03) *$ & $<0.001^{*}$ \\
Duration of inotropes & $1.37 \pm 1.27$ & $2.61 \pm 3.17$ & $1.51(1.23-1.84)^{*}$ & $<0.001^{*}$ \\
Post-op stroke & $4(0.8)$ & $3(3.3)$ & $4.42(0.97-20.10)$ & $0.036^{*}$ \\
Post-op renal dysfunction & $2(0.4)$ & $0(0)$ & 0 & 0.555 \\
Length of ICU stay & $2.95 \pm 1.89$ & $5.32 \pm 4.37$ & $1.50(1.30-1.74) *$ & $<0.001^{*}$ \\
Length of hospital stay & $10.54 \pm 4.34$ & $13.81 \pm 8.81$ & $1.09(1.05-1.13) *$ & $<0.001^{*}$ \\
30-day mortality & & & & $0.005^{*}$ \\
$\quad$ Alive & $525(99.2)$ & $88(95.7)$ & $5.97(1.47-24.29)$ & \\
\hline Dead & $4(0.8)$ & $4(4.3)$ & & \\
\hline
\end{tabular}

Significant $p$ value $<0.05^{*}$ and $<0.001 * *$

$n$ number of patients, NSR normal sinus rhythm, $A F$ atrial fibrillation, post-op post-operative, $I C U$ intensive care unit, $O R$ odds ratio, $C I$ confidence interval which alters electrophysiological properties of atria, namely, shortness of effective refractory period, dispersion of refractoriness and conduction, abnormal automaticity, and anisotropic conduction, which predisposes to development of AF [10]. One study showed that [10] for every 10-year increase in age, there was a $75 \%$ increase in the chance for developing AF. We also found significant increase in POAF occurrence with each passing decade after 50 years: $1.8 \%$ in patients < 50 years, $10.79 \%$ in $50-59$ years, $19.25 \%$ in $60-69$ years, $28.22 \%$ in $70-79$ years, and $50 \%$ in patients above the age of 80 years.

Sepsis after OPCAB is thought to be less due to avoidance of the inflammatory response of CPB. However, surgical stresses, blood transfusions, and endotoxemia from bacterial translocation from gut are some factors that provoke the development of systemic inflammatory syndrome/sepsis in OPCAB [24]. In our series, $11.2 \%$ of patients developed clinical and or laboratory features suggestive of sepsis. $2.67 \%$ had positive bacterial cultures. It is also likely that secondary to multiple systemic issues in healthcare, the burden of

Table 6 Follow-up of low-risk patients

\begin{tabular}{lll}
\hline Variables & \multicolumn{2}{l}{ Alive $(n=595)$} \\
\cline { 2 - 3 } & $\begin{array}{ll}\text { NSR }(n(\%)) \\
509(\%)\end{array}$ & $\begin{array}{l}\text { AF }(n(\%)) \\
86(\%)\end{array}$ \\
\hline Revascularization & $9(1.77)$ & $2(2.32)$ \\
Stroke & $2(0.39)$ & $2(2.32)$ \\
Heart failure & $2(0.39)$ & $0(0)$ \\
Renal dysfunction & $11(2.16)$ & $6(6.98)$ \\
Renal failure requiring dialysis & $3(0.59)$ & $0(0)$ \\
Myocardial infarction & $5(0.98)$ & $0(0)$ \\
Angina & $7(1.37)$ & $2(2.32)$ \\
Mid-term mortality & $9(1.77)$ & $2(2.32)$ \\
\hline
\end{tabular}

NSR normal sinus rhythm, $A F$ atrial fibrillation, $n$ number of patients nosocomial sepsis, including post-operative sepsis, is higher in emerging economies. In multivariate analysis, it has emerged as a strong risk factor for development of POAF. Systemic inflammation associated with sepsis is a wellknown trigger for development of POAF in critically ill patients [25]. This study highlights the important contribution of sepsis to the occurrence of POAF, hitherto probably under reported in the post-operative cardiac surgical population. POAF will worsen the hemodynamic parameters in patients with both diastolic dysfunction [26] and systolic dysfunction emphasizing needs for the health systems in these countries to target sepsis control as high priority. All these factors potentially lead to prolonged ICU stay with increased mortality.

Ventilator hours, length of ICU stay, and length of hospital stay were increased in POAF patients. ICU stay was increased by 1.5 days and hospital stay by 1 day and ventilator hours by $20 \mathrm{~h}$ in POAF patients. Stroke is a devastating complication after cardiac surgery. The stroke rate was $3.1 \%$ in patients developing POAF compared with $0.8 \%$ in NSR (fourfold increase in POAF). Moreover, there was a tendency for increased stroke rate on follow-up in POAF patients. POAF is a well-known cause of late recurrence of AF with potential hazard for stroke. These results were independent of the risk profile of patients.

In our study, there was a fivefold increase in 30-day mortality in patients with POAF. This trend of decreased survival continued in the follow-up period. It is still not clear in existing literature whether the increased risk for mortality in POAF patients is due to the effect of AF or whether AF is only a surrogate marker for severity of illness [27-31]. Further, the independent contribution of POAF to these greatly increased risks is unknown as sepsis, in itself, can hugely impair postoperative outcome. The analysis of the impact of POAF in the low-risk cohort underlines the need for emphasizing its impact on overall outcomes, and, in turn, to develop a strategy for prevention as a key quality improvement measure. 
Fig. 2 A and B Kaplan-Meir estimate of survival of patients in low-risk group NSR vs. POAF

Survival Curve and Hazard function in low risk group

a

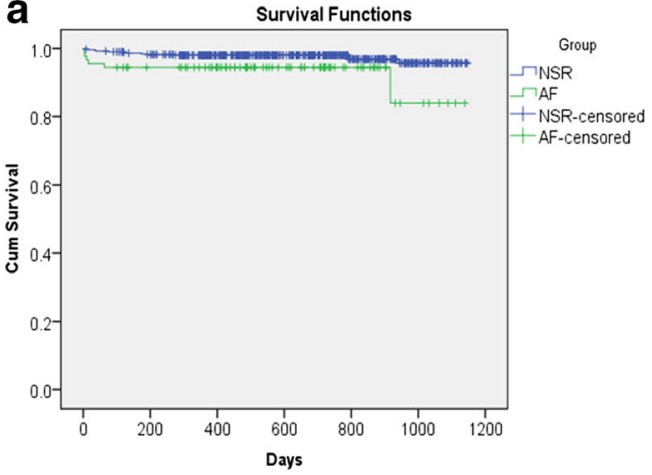

b

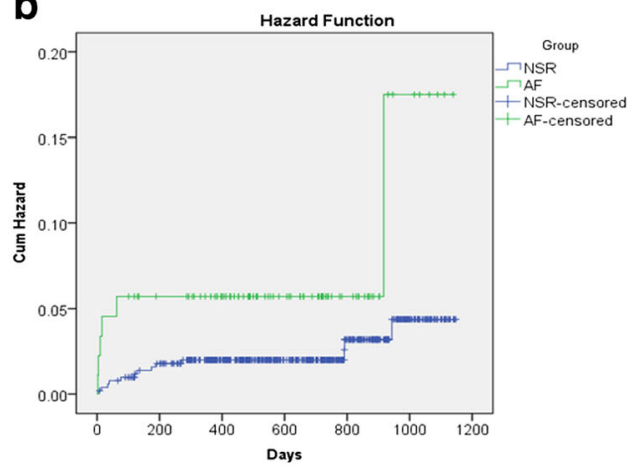

\section{Study limitations}

This is a single-center study. We examined only 12 preoperative variables for risk analysis. Therefore, other predictors associated with POAF could have been excluded. Continuous ECG monitoring of patients occurred only during ICU stay. More systematic ECG monitoring could result in a better identification and a higher detected incidence of AF. We also do not have any data regarding occurrence of late AF. It is clinically difficult to separate sepsis due to infection and systemic inflammatory response. Hence, we used the STS definition to document sepsis. The patients in the medium- and high-risk categories (EuroSCORE $>3$ and 6 respectively) were small in number in the overall POAF cohort, limiting analysis for these patients. The study however is likely the first study with complete immediate follow-up and $97 \%$ follow-up over the midterm (follow-up of 2 years) from the Indian subcontinent. The report will hopefully spurn multicenter initiatives to map surgical outcomes and collaborative preventive and quality improvement measures.

\section{Conclusion}

POAF remains a common complication after CABG; however, the incidence seemed to be lower than reported in western literature. Increasing age and development of post-operative sepsis were strong independent predictors of POAF. The standard measurable outcomes like mortality, stroke, and renal failure requiring dialysis, ventilator hours, length of ICU stay, and length of hospital stay were increased in patients developing POAF. However, sepsis remains a confounding variable and may be the trigger for development of POAF and the increase in adverse events. There should be a renewed interest in developing effective preventive strategies for the occurrence of POAF. Those patients who develop POAF should be followed up meticulously to reduce complications and improve the survival in the long term.
Funding information The authors received no financial support for the research, authorship, and /or publication of this article.

\section{Compliance with ethical standards}

Conflict of interest The authors declare that they have no conflict of interest.

Ethical approval The study was approved by the hospital ethics committee. The need for informed consent was waived off. All personal identifying data was removed from the study database so that individuals could not be identified. "All procedures performed in studies involving human participants were in accordance with the ethical standards of the institutional and/or national research committee and with the 1964 Helsinki declaration and its later amendments or comparable ethical standards."

Informed consent For this type of study, formal consent is not required.

\section{References}

1. Aranki SF, Shaw DP, Adams DH, et al. Predictors of atrial fibrillation after coronary artery surgery. Current trends and impact on hospital resources. Circulation. 1996;94:390-7.

2. Peretto G, Durante A, Limite LR, Cianflone D. Postoperative arrhythmias after cardiac surgery: incidence, risk factors, and therapeutic management. Cardiol Res Pract. 2014. https://doi.org/10. 1155/2014/615987.

3. Hogue CW Jr, Creswell LL, Gutterman DD, Fleisher LA, American College of Chest Physicians. Epidemiology, mechanisms, and risks: American College of Chest Physicians guidelines for the prevention and management of postoperative atrial fibrillation after cardiac surgery. Chest. 2005;128:9S-16S.

4. Echahidi N, Pibarot P, O'Hara G, Mathieu P. Mechanisms, prevention, and treatment of atrial fibrillation after cardiac surgery. J Am Coll Cardiol. 2008;51:793-801.

5. Salaria V, Mehta NJ, Abdul-Aziz S, Mohiuddin SM, Khan IA. Role of postoperative use of adrenergic drugs in occurrence of atrial fibrillation after cardiac surgery. Clin Cardiol. 2005;28:131-5.

6. Cox JL. A perspective of postoperative atrial fibrillation in cardiac operations. Ann Thorac Surg. 1993;56:405-9.

7. Ascione R, Caputo M, Calori G, Lloyd CT, Underwood MJ, Angelini GD. Predictors of atrial fibrillation after conventional 
and beating heart coronary surgery: A prospective, randomized study. Circulation. 2000;102:1530-5.

8. Wan S, Izzat MB, Lee TW, Wan IY, Tang NL, Yim AP. Avoiding cardiopulmonary bypass in multivessel $\mathrm{CABG}$ reduces cytokine response and myocardial injury. Ann Thorac Surg. 1999;68:52-6.

9. Borde D, Gandhe U, Hargave N, Pandey K, Mathew M, Joshi S. Prediction of postoperative atrial fibrillation after coronary artery bypass grafting surgery: Is CHA2DS2-VASc score useful? Ann Card Anaesth. 2014;17:182-7.

10. Mathew JP, Fontes ML, Tudor IC, et al. A multicenter risk index for atrial fibrillation after cardiac surgery. JAMA. 2004;291:1720-9.

11. Loubani M, Hickey MS, Spyt TJ, Galiñanes M. Residual atrial fibrillation and clinical consequences following postoperative supraventricular arrhythmias. Int J Cardiol. 2000;74:125-32.

12. Pfisterer ME, Kloter-Weber UC, Huber M, et al. Prevention of supraventricular tachyarrhythmias after open heart operation by low-dose sotalol: a prospective, double-blind, randomised, placebo-controlled study. Ann Thorac Surg. 1997;64:1113-9.

13. Mariscalco G, Klersy C, Zanobini M, et al. Atrial fibrillation after isolated coronary surgery affects late survival. Circulation. 2008;118:1612-8.

14. Filardo G, Hamilton C, Hebeler RF Jr, Hamman B, Grayburn P. New-onset postoperative atrial fibrillation after isolated coronary artery bypass graft surgery and long-term survival. Circ Cardiovasc Qual Outcomes. 2009;2:164-9.

15. Kaireviciute D, Aidietis A, Lip GY. Atrial fibrillation following cardiac surgery: Clinical features and preventative strategies. Eur Heart J. 2009;30:410-25.

16. LaPar DJ, Speir AM, Crosby IK, et al. Postoperative atrial fibrillation significantly increases mortality, hospital readmission, and hospital costs. Ann Thorac Surg. 2014;98:527-3.

17. Lazar HL. Should Off-Pump Coronary Artery Bypass Grafting Be Abandoned? Circulation. 2013;128:406-13.

18. Mehta RL, Kellum JA, Shah SV, et al. Acute Kidney Injury Network: report of an initiative to improve outcomes in acute kidney injury. Crit Care. 2007;11:R31.

19. D'Agostino RS, Jacobs JP, Badhwar V, et al. The Society of Thoracic Surgeons Adult Cardiac Surgery Database: 2018 Update on Outcomes and Quality. Ann Thorac Surg. 2018;105:15-23.

20. Shroyer AL, Grover FL, Hattler B, et al. On-pump versus off-pump coronary-artery bypass surgery. N Engl J Med. 2009;361:1827-37.

21. Hueb W, Lopes NH, Pereira AC, et al. Five-year follow-up of a randomized comparison between off-pump and on-pump stable multivessel coronary artery bypass grafting. The MASS III Trial Circulation. 2010;122:S48-52.

22. Akintoye E, Sellke F, Marchioli R, Tavazzi L, Mozaffarian D. Factors associated with postoperative atrial fibrillation and other adverse events after cardiac surgery. J Thorac Cardiovasc Surg. 2018;155:242-51.

23. Varma PK. Prediction of postoperative atrial fibrillation after cardiac surgery: Light at the end of the tunnel? Ann Card Anaesth. 2014;17:187-90.

24. Gomes WJ, Erlichman MR, Batista-Filho ML, et al. Vasoplegic syndrome after off-pump coronary artery bypass surgery. Eur $\mathrm{J}$ Cardiothorac Surg. 2003;23:165-9.

25. Chelazzi C, Giugni D, Villa G, De Gaudio A. Postoperative atrial fibrillation and sepsis: 12AP3-5. Eur J Anaesthesiol. 2012;29:181.

26. Rosenberg MA, Manning WJ. Diastolic Dysfunction and Risk of Atrial Fibrillation: A Mechanistic Appraisal. Circulation. 2012;126: 2353-62.
27. Melduni RM, Schaff HV, Bailey KR, et al. Implications of newonset atrial fibrillation after cardiac surgery on long-term prognosis: A community-based study. Am Heart J. 2015;170:659-68.

28. Villareal RP, Hariharan R, Liu BC, et al. Postoperative atrial fibrillation and mortality after coronary artery bypass surgery. J Am Coll Cardiol. 2004;43:742-8.

29. Mariscalco G, Engstrom KG. Postoperative atrial fibrillation is associated with late mortality after coronary surgery, but not after valvular surgery. Ann Thorac Surg. 2009;88:1871-6.

30. Almassi GH, Pecsi SA, Collins JF, Shroyer AL, Zenati MA, Grover FL. Predictors and impact of postoperative atrial fibrillation on patients' outcomes: A report from the Randomized on Versus off Bypass trial. J Thorac Cardiovasc Surg. 2012;143:93-102.

31. Ad N, Massimiano PS. Postoperative atrial fibrillation: Adding (fish) oil to the fire. J Thorac Cardiovasc Surg. 2018;155:252-3.

\section{Discussant:}

Dr. Surendra K Agarwal,

Professor, Department of CVTS,

SGPGIMS, Lucknow 226 014, India

1. It is a well conducted study but details only about off pump CABG. As there were some patients which were done on-pump (25, excluded from the study). Can the authors tell about the incidence of AF in these patients, if any?

Ans: We did not specifically look into these data because the numbers were small and this group of patients were different from the routine patients done on pump; many were conversions both elective and emergency and some had elective LAD end arterectomy.

2. The anticoagulation was stopped if NSR was restored. What was the followup protocol for Amiodarone? This will be useful information for the readers. Ans: Amiodarone was continued for 3 months once initiated

3. How was the rhythm decided in patients who were contacted telephonically?

Ans: Telephonic follow up was done for determining the major adverse events. We did not look into the recurrence of $\mathrm{AF}$ in any patients.

4. Authors have given a median follow-up of 630 days. Data on mean with standard deviations would have been more meaningful for this study with a good sample size. What were the number and percentage of patients available for follow-up at 2 and 3 years followup?

Ans: We do agree with first suggestion. Regarding the number of patients: In 2yr- 280 (37.4\%) patients; 3yr- 20(2.7\%) patients were available for follow up. 5. The authors could have done a multivariate analysis to determine the effect of other risk factors like sepsis, IABP use etc. on the mortality to provide a realistic effect of AF on short and intermediate term mortality. Any data or your thoughts?

Ans: Since the number of dead patients was low, we thought it may be inappropriate to do multivariate analysis to determine the effect of other risk factors like sepsis, IABP use etc on mortality.

6. The last is just a suggestion. As the authors have followed these patients, they may continue to follow these patients for another 5-10 years to get the effect of $\mathrm{AF}$ on long term mortality.

Ans: Thank you for the suggestion, we will try to do this.

Publisher's note Springer Nature remains neutral with regard to jurisdictional claims in published maps and institutional affiliations. 